\title{
Innovative Applications of Artificial Intelligence Techniques in Software Engineering
}

\author{
Masoud Mohammadian \\ University of Canberra, ACT, Australia \\ Masoud.mohammadian@canberra.edu.au
}

\begin{abstract}
Artificial Intelligence (AI) techniques have been successfully applied in many areas of software engineering. The complexity of software systems has limited the application of AI techniques in many real world applications. This talk provides an insight into applications of AI techniques in software engineering and how innovative application of AI can assist in achieving ever competitive and firm schedules for software development projects as well as Information Technology (IT) management. The pros and cons of using AI techniques are investigated and specifically the application of AI in IT management, software application development and software security is considered.

Organisations that build software applications do so in an environment characterised by limited resources, increased pressure to reduce cost and development schedules. Organisations demand to build software applications adequately and quickly. One approach to achieve this is to use automated software development tools from the very initial stage of software design up to the software testing and installation. Considering software testing as an example, automated software systems can assist in most software testing phases.

On the hand data security, availability, privacy and integrity are very important issues in the success of a business operation. Data security and privacy policies in business are governed by business requirements and government regulations. AI can also assist in software security, privacy and reliability. Implementing data security using data encryption solutions remain at the forefront for data security. Many solutions to data encryption at this level are expensive, disruptive and resource intensive. AI can be used for data classification in organizations. It can assist in identifying and encrypting only the relevant data thereby saving time and processing power. Without data classification organizations using encryption process would simply encrypt everything and consequently impact users more than necessary. Data classification is essential and can assist organizations with their data security, privacy and accessibility needs. This talk explores the use of AI techniques (such as fuzzy logic) for data classification and suggests a method that can determine requirements for classification of organizations' data for security and privacy based on organizational needs and government policies. Finally the application of FCM in IT management is discussed.
\end{abstract}

